Host zum istrischen $D$. ciliatus, wie schon vordem $\mathrm{Koch}$ gethan hatte.

Mithin ist erwiesen, dass D. ciliatus Guss., D. racemosus Vis., $D$. ciliatus $\alpha$. racemosus Vis., $D$. litoralis Host Synonyme sind, und dass der $D$. ciliatus $\beta$. cymosus Vis. (exclus. synon. $D$. litoralis Host) als eigene Art, welcher der irrige Beiname cymosus nicht boigelegt werden kann, einen neuen Namen erhalten musste.

\title{
Zur Kenntniss der Kryptogamenflora von Tirol.
}

\section{Von Dr. D. C. Schiedermayr. $\left.{ }^{1}\right)$}

\section{Algen.}

Spirogyra areta Kï̈̈tz. Im Bassin des Klosterbrunnens zu St. Georgenberg bei Schwaz, conjugirt.

Ulothrix radicans Kütz. An entblössten Baumwurzeln im Volderthale.

- zonata Ktzg. Mit Spirogyra arcta zu St. Georgenberg.

Zygnema affine Ktzg. Mit der vorigen.

\section{Pilze.}

Aecidium Euphorbiae Gmel. Anf Euphorbia Cyparissias im Aufstiege von Hall zum Volderbade.

Ascobolus granulatus (Bull) Fuck. Auf Kuhmist im Volderthale und beim Goldbrünnl nächst Windegg.

Boletus subtomentosus L. In Wäldern des Volderthales.

Chrysomyxa Rhododendri (DC.). Auf der Unterseite der Blätter von Rhododendron ferrugineum in den Voralpen im Volderthale.

Melampsora Vaccinii (Alb. et Schw.). Auf der Unterseite der Blätter von Vaccinium Myrtillus in Wäldern des Volderthales.

- Goeppertiana (Kühn) Wint. An den Stämmchen von Vaccinium Vitis idaea bei Hall.

Peronospora pusilla Ung. Auf Blättern von Geranium silvatioum auf

Wiesen bei der Stifts- (Unterleger-) Alpe.

Polyporus abietinus Fr. Auf Fichtenstrünken im Volderthale.

Puccinia Anemones virginianae Schweinitz. Auf der Unterseite eines Blattes von Atragene alpina im Volderthale.

- Flosculosorum (Alb. et Schw.). Var. Hieracii Schumacher. Die

1) Sämmtlich gesammelt rom 26. Juni bis 8 . Juli 1884 während meines Aufenthaltes im Bade Volderthal bei Hall, wo auch ein Theil der von Ds: Leithe im laufenden Jahrgange unserer Zeitschrift verzeichneten Arten gesammelt wurde. Um daher nicht bereits Mitgetheiltes za geben, sind in diesem Verzeichnisse nur jene Arten aufgenommen, welche im Verzeichnisse Dr. Leithe's nicht vorkommen. 
Teleutosporenform anf Hieracium murorum in Wäldern des Volderthales.

Puccinia Prenanthis (Pers.). Dio Aecidienform auf Prenanthes muralis in Wäldern im Volderthal.

Ramularia macrospora Sacc. Auf Blättern von Phyteuma betonicaefolium an Waldrändern im Volderthal.

Septoria Violae Rbh. (vide Fuckel, Symb. mycolog. II. Nachtrag pag. 82.) Auf der Oberseite der Blätter von Viola biflora im Volderthale.

Uredo alpestris Schroet. Auf der Unterseite der Blätter von Viola biflora im Volderthale.

Uromyces Geranii (DC.). Die Aecidienform auf Geranium silvaticum auf Alpenwiesen der Stifts- (Unterleger-) Alpe.

Ustilago violacea DC. In den Antheren von Silene rupestris im Volderthale.

\section{Flechten.}

Aspicilia cinerea (L.) Körb. Auf Phyllitblöcken am Schwarzbrunn.

Biatora granulosa (Ehrh.) Fr. In den Fugen alter Steindämme unterhalb der Stifts- (Unterleger-) Alpe.

- lucida (Ach.) Fr. Auf Phyllit-Trümmern alter Steindämme wie oben.

Cladonia furcata (Huds.) Hoffm. Var. subulata (L.) Fl. Casp. Ueber Phyllit auf Waldboden im Volderthale.

- rangiformis Hoffm. Wie die vorige.

- uncialis (L.) Hoffm. Auf dürren, lichten Waldstellen unterhalb der Stifts- (Unterleger-) Alpe.

Cetraria cucullata (L.) Ach. Auf dürrem Boden auf dem Tulfeiner Jöchl.

- nivalis (L.) Ach. Mit der vorigen.

Evernia vulpina (L.) Ach. An der entblössten Rinde abgestorbener Zirbelkiefern auf dem Tulfeiner Jöchl.

Gyrophora polyphylla (L.) Ach. Ueber Phyllit auf dem Glungezer. Lecidea macrocarpa (DC.). Ueber Phyllit auf dem Glungezer und beim Schwarzbrunn.

Leptogium lacerum (Sw.). Auf bemoosten Phyllitblöcken im Volderthale.

Ramalina pollinaria Ach. Auf Phyllit-Trümmern eines Steindammes unterhalb der Stifts- (Unterleger-) Alpe.

Rhizocarpon geographicum (L.) DC. Auf Phyllit vom Thale bis in die Alpen, häufig.

Solorina saccata (L.) Ach. Ueber Phyllit im Volderthale.

Urceolaria scruposa Ach. var. bryophila. Auf Moospolstern über Phyllit im Volderthale.

\section{Lebermoose.}

Jungermannia trichophylla L. Auf morschen Baumstrünken im Volderthale, durchwachsen mit Lepidozia reptans. 


\section{Laubnoose.}

Ceratodon purpureus (L.) Brid. Auf Phyllitblöcken am Schwarzbrunn. Fissidens adianthoides Hedw. An quelligen Orten im Volderthale. Grimmia Mühlenbeckii Schimp. Auf Phyllitblöcken im Volderthale. Hypnum molluscum Hedw. Auf Kalkfelsen in der Placidus-Klause za St. Georgenberg bei Schwaz.

Mnium punctatum Hedw. An quelligen Orten im Volderthale.

- serratum Brid. Wie das vorige.

Trichostomum crispulum Bruch. In lehmichten Hohlwegen im Volderthale.

Zieria julacea Schimp. Mit Ceratodon purpureus gemengt auf Phyllitblöcken am Schwarzbrunn.

\section{Gefässkryptogamen.}

Equisetum silvaticum L. An Waldrändern im Volderthale. Phegopteris polypodioides Fee. Häufig in Wäldern im Volderthale.

\section{Teratologisches.}

\section{Von Prof. Fr. Vierhapper.}

Im Hefte Nr. 3 der Oest. bot. Zeitschr. Jahrg. XXXV (1885), ist ein Aufsatz von Wilhelm Voss: "Eine seltene Bildungsabweichung an der Frühlingsknotenblume "Lewcojum vernum L.)" enthalten. - Aus der Umgebung von Ried wurde mir im März des vorigen Jahres ein Leucojum vernum L. gebracht, welches eine ganz ähnliche Zwiebelverdopplung zeigte. Das mir überbrachte Exemplar stimmt am meisten mit dem von Voss als 2. Fall beschriebenen überein. Nur trug es eine vollkommen entwickelte Blüthe, die sich von der einer normal entwickelten Pflanze nicht im geringsten unterschied und genau dieselben Grössenverhältnisse aufwies. Auch heuer wurde an einer ganz anderen Stelle ein Leucojum mit ganz ähnlicher abnormer Zwiebelbildung aufgefunden, so dass diese merkwürdige Erscheinung vielleicht öfter vorhanden ist, als man bisher vermuthete, und nur so selten beobachtet wurde, weil in der Regel bei dem geselligen Vorkommen dieser Pflanze verbältnissmässig doch nur wenige Exemplare ausgegraben werden. Eine genügende Erklärung dieser abweichenden Zwiobelentwicklung zu geben, ist sehr schwierig, da eine oder zwei Beobachtungen nicht hinreichen, sich ein klares Urtheil zu bilden. Den Fall im vorigen Jahre babe ich etwas eingehender untersuchen können. Die Pflanze stand an einer sehr feuchten, mehr schlammigen Stelle, und die unterste, offenbar zuerst gebildete Zwiebel war sehr tief in den Boden eingesenkt und zwar tiefer, als diess bei gewöhnlichen Leucojum-Exemplaren, selbst von üppigster Vegetation zu sein pflegt. Die Pflanze hat von der untersten Zwiebel bis zur höchsten Stelle des Schaftes eine unge- 